\title{
Sobczyk's theorem and the Bounded Approximation Property
}

\author{
by \\ Jesús M. F. Castillo and Yolanda Moreno (Badajoz)
}

\begin{abstract}
Sobczyk's theorem asserts that every $c_{0}$-valued operator defined on a separable Banach space can be extended to every separable superspace. This paper is devoted to obtaining the most general vector valued version of the theorem, extending and completing previous results of Rosenthal, Johnson-Oikhberg and Cabello. Our approach is homological and nonlinear, transforming the problem of extension of operators into the problem of approximating $z$-linear maps by linear maps.
\end{abstract}

1. Introduction. Sobczyk's theorem $[\underline{S}]$ is one of the fundamental theorems in Banach space theory. Its simplest formulation, omitting quantitative estimates, is perhaps the following:

SobczYK's Theorem. Given a closed subspace $Y$ of a separable Banach space $Z$, every operator $\tau: Y \rightarrow c_{0}$ admits an extension $T: Z \rightarrow c_{0}$.

In spite of its importance, the only well known aspect of this result is the scalar valued separable case. Indeed, the papers [ACGJM, GP, KZ] explore the possibilities and limitations of scalar valued nonseparable versions; while $[\mathrm{C}, \mathrm{JO}, \mathrm{R}$, consider a vector valued separable case. The interested reader is referred to $\mathrm{CCY}$, where a fairly complete survey of all the proofs of Sobczyk's theorem appearing in the literature until 2000 is presented, including a nonlinear proof close in spirit to the approach in this paper.

One of the basic open questions about this result is to what extent it remains true on replacing functionals by operators; or, if one prefers, on replacing as target space the scalar field by an arbitrary Banach space. This paper solves the problem by showing that an optimal vector valued version of Sobczyk's theorem holds if and only if the quotient space has the Bounded Approximation Property. Let us explain the meaning of "optimal version".

2010 Mathematics Subject Classification: Primary 46M18, 46B25, 46T99; Secondary $46 \mathrm{~B} 28$.

Key words and phrases: extension and lifting of operators, separable injectivity, Bounded Approximation Property, twisted sums of Banach spaces. 
Observe that an operator $\tau: Y \rightarrow c_{0}\left(E_{n}\right)$ corresponds to a sequence of operators $t_{n}: Y \rightarrow E_{n}$ with the property, called sometimes "being SOT-null" that $\lim \left\|T_{n} y\right\|=0$ for each $y \in Y$. There is no loss of generality in assuming that all spaces $E_{n}$ are the same space $E$, since one can always replace each $E_{n}$ by $E=c_{0}\left(E_{n}\right)$. When $E$ is the scalar field, a SOT-null sequence of operators $Y \rightarrow E$ is actually a weak ${ }^{*}$-null sequence of functionals on $Y$. Hence, the classical Sobczyk's theorem can be rephrased as: Given a subspace $Y$ of a separable Banach space $Z$, a weak $k^{*}$-null sequence of continuous linear functionals on $Y$ admits a weak ${ }^{*}$-null sequence of extensions to $Z$; and thus a straight vector valued translation could be: Given a subspace $Y$ of a separable Banach space Z, a SOT-null sequence of operators $Y \rightarrow E$ admits a SOTnull sequence of extensions to $Z$. However, this cannot be true because an obvious necessary condition to get a SOT-null sequence of extensions is the mere existence of a uniformly bounded sequence of extensions, which means that every operator $Y \rightarrow E$ can be extended to an operator $Z \rightarrow X$. A Banach space $E$ with this property is said to be separably injective; namely, $E$-valued operators defined on separable spaces admit extensions to separable superspaces. Hence, a necessary condition to obtain the straight result quoted above is that $E$ is separably injective. This is also a sufficient condition since one has

Proposition 1.1. If $E$ is a separably injective space then $c_{0}(E)$ is separably injective.

This result has been independently obtained by Rosenthal in [R], using operator techniques, by Johnson and Oikhberg in [JO], using the theory of M-ideals, and by Cabello [C] using a topological approach. The class of separably injective Banach spaces is not narrow and in fact contains interesting members, some quite exotic: all injective spaces, the spaces $c_{0}(I)$, twisted sums and quotients of two separably injective spaces etc.; there even exist [CMS, ACCGM] separably injective spaces which are not complemented subspaces of a $C(K)$-space. The monograph [ACCGM] contains a thorough study of this class. Nevertheless, we want to handle a broader situation in which one does not require $E$ to be separably injective. Indeed, the situation we want to handle is one where $E$ is only required to have one specific SOTnull sequence of operators $\tau_{n}: Y \rightarrow E$ that admits a uniformly bounded sequence of extensions $Z \rightarrow E$ through one specific embedding $j: Y \rightarrow Z$. In that case, does there also exist a SOT-null sequence of extensions? What we will do is to remove the "universality" condition on the space $E$ at the cost of imposing a reasonable hypothesis on the quotient space $X=Z / Y$ : to have the Bounded Approximation Property. Recall that a separable Banach space $X$ is said to enjoy the $\lambda$-Bounded Approximation Property, for short $\lambda$-BAP, if there exists a sequence $B_{n}: X \rightarrow X$ of finite-dimensional linear 
operators with norms $\left\|B_{n}\right\| \leq \lambda$ that is pointwise convergent to the identity. A Banach space is said to have the Bounded Approximation Property, for short BAP, if it has the $\lambda$-BAP for some $\lambda$. One then has:

Theorem 1.2 (Vector valued Sobczyk theorem: extension version). Let $Y$ be a subspace of a Banach space $Z$ such that $Z / Y$ is separable and has the Bounded Approximation Property. Then every SOT-null sequence of operators on $Y$ that admits a uniformly bounded sequence of extensions to $Z$ also admits a SOT-null sequence of extensions to $Z$.

Our approach to this result is a combination of homological and nonlinear techniques. We first use the representation of exact sequences of Banach spaces by means of $z$-linear maps (see [CG, CC1, CM], or the next section of this paper). The space of $z$-linear maps carries a natural seminorm, to which the term "uniformly" refers from now on. Next, the extension problem for operators is transformed into the problem of approximating $z$-linear maps by linear maps. If we define a $z$-linear map to be trivial when it can be approximated by a linear map then the nonlinear version of the vector valued form of Sobczyk's theorem essentially says that if $\left(F_{n}\right)$ is a pointwise null sequence of (uniformly) trivial $z$-linear maps defined on a space with the BAP then the $z$-linear map $c_{0}\left(F_{n}\right)$ is trivial. Precisely

Theorem 1.3 (Vector valued Sobczyk theorem: nonlinear version). Let $X$ be a separable Banach space with the BAP. Let $F_{n}: X \curvearrowright E_{n}$ be a uniformly bounded sequence of $z$-linear maps such that $\lim \left\|F_{n} x\right\|=0$ for every $x \in X$. If all the maps $F_{n}$ are uniformly trivial then the map $c_{0}\left(F_{n}\right)$ is trivial.

Observe that it follows from the uniform boundedness principle that a SOT-null sequence of operators is uniformly bounded, while a pointwise null sequence of $z$-linear maps must be assumed to be uniformly bounded. To prove this version we develop a "chasing device"-inspired by Veech's proof of Sobczyk's theorem - that is flexible enough to adapt to other situations, as can be seen in the "Applications" section. Moreover, the chasing device evidences that an optimal extension result for the extension of operator $Y \rightarrow$ $c_{0}(E)$ to operators $Z \rightarrow c_{0}(E)$ is obtained by a combination of properties of $Z / Y$ and $E$ : asking nothing of $E$ requires that $Z / Y$ (be separable and) have the BAP; asking everything of $E$ (namely, to be separably injective) requires only the separability $Z / Y$; etc. Proposition 5.3 provides still another striking example.

Quantitative estimates. There are quantitative estimates involved in the previous results, all of them related to the ratio between the norm of the extended operator $Z \rightarrow c_{0}(E)$ and of the original one $Y \rightarrow c_{0}(E)$. A Banach space $E$ is said to be $\lambda$-separably injective if every norm one $E$-valued oper- 
ator admits an extension to any separable superspace with norm at most $\lambda$. It is obvious that every separably injective space is $\lambda$-separably injective for some $\lambda$. Proposition 1.1 becomes, in quantitative terms, the question of obtaining a function $f(\lambda)$ for which $c_{0}(E)$ is $f(\lambda)$-separably injective when $E$ is $\lambda$-separably injective. Our estimate for $f(\lambda)$ is of order $6 \lambda$ (see Thm. 4.5), which asymptotically improves all previous results: $2 \lambda^{2}$ of Johnson-Oikhberg [JO (implicit); $\lambda(1+\lambda)$ of Rosenthal [R]; and $3 \lambda^{2}$ of Cabello [C]. We will return to this point later.

2. Preliminaries. An exact sequence $0 \rightarrow Y \rightarrow Z \rightarrow X \rightarrow 0$ in the category of Banach spaces and continuous linear operators is a diagram in which the kernel of each arrow coincides with the image of the preceding one; the middle space $Z$ is also called a twisted sum of $Y$ and $X$. By the open mapping theorem, an exact sequence as above means that $Y$ is a subspace of $Z$ and $X$ is the corresponding quotient. An exact sequence is said to split if it is equivalent to the trivial sequence $0 \rightarrow Y \rightarrow Y \oplus X \rightarrow X \rightarrow 0$.

There is a correspondence (see [K1, $\mathrm{KP}, \mathrm{CG}$ ) between exact sequences $0 \rightarrow Y \rightarrow Z \rightarrow X \rightarrow 0$ of Banach spaces and the so-called z-linear maps which are homogeneous maps $F: X \curvearrowright Y$ (we use this notation to stress that they are not linear) with the property that there exists some constant $C>0$ such that for all finite sets $\left\{x_{1}, \ldots, x_{n}\right\} \subset X$ one has

$$
\left\|F\left(\sum_{n=1}^{N} x_{n}\right)-\sum_{n=1}^{N} F\left(x_{n}\right)\right\| \leq C \sum_{n=1}^{N}\left\|x_{n}\right\| .
$$

The infimum of the constants $C$ is called the $z$-linearity constant of $F$ and denoted $Z(F)$. To obtain a $z$-linear map from an exact sequence $0 \rightarrow Y \rightarrow$ $Z \stackrel{q}{\rightarrow} X \rightarrow 0$, take a homogeneous bounded selection $b: X \rightarrow Z$ for the quotient map $q$, and then a linear selection $\ell: X \rightarrow Z$ for the quotient map (in particular, see the linearization process below). Then $F=b-\ell$ is a $z$-linear map. Consequently, a z-linear map $F: X \curvearrowright Y$ induces the exact sequence of quasi-Banach spaces $0 \rightarrow Y \rightarrow Y \oplus_{F} X \rightarrow X \rightarrow 0$ in which $Y \oplus_{F} X$ means the vector space $Y \times X$ endowed with the quasi-norm $\|(y, x)\|_{F}=\|y-F x\|+\|x\|$.

If the embedding $Y \rightarrow Z$ is an into isometry and $q$ the corresponding quotient operator then the quotient map $Z \rightarrow X$ admits a homogeneous bounded selection $b: X \rightarrow Z$ with $\|b\| \leq 1$. The sequences $0 \rightarrow Y \rightarrow Z \rightarrow$ $X \rightarrow 0$ and $0 \rightarrow Y \rightarrow Y \oplus_{F} X \rightarrow X \rightarrow 0$ are equivalent and the operator $T: Y \oplus_{F} X \rightarrow Z$ given by $T(y, x)=y+\ell x$ establishes the equivalence. Moreover, $\|T\| \leq 1$ and $\left\|T^{-1}\right\| \leq 3$ :

$$
\begin{gathered}
\|T(y, x)\|=\|y-F x+F x+\ell x\| \leq\|y-F x\|+\|b x\| \leq\|(y, x)\|_{F}, \\
\left\|T^{-1} z\right\|=\|z-\ell q z-F q z\|+\|q z\|=\|z-b q z\|+\|q z\| \leq 3\|z\| .
\end{gathered}
$$


The map $F$ is $z$-linear if and only if $\|\cdot\|_{F}$ is equivalent to a norm (see [CA]). Let $\operatorname{co}\left(Y \oplus_{F} X\right)$ be the Banach envelope of $Y \oplus_{F} X$, i.e., the Banach space whose unit ball is the closed convex envelope of the unit ball of $\|\cdot\|_{F}$. The spaces $\operatorname{co}\left(Y \oplus_{F} X\right)$ and $Y \oplus_{F} X$ are $Z(F)+1$-isomorphic. Two $z$-linear maps $F, G: X \curvearrowright Y$ are said to be equivalent, written $F \equiv G$, if the induced exact sequences are equivalent (for the classical homological equivalence of exact sequences). Two maps $F, G: X \curvearrowright Y$ are equivalent if and only if the difference $F-G$ can be written as $B+L$, where $B: X \rightarrow Y$ is a homogeneous bounded map and $L: X \rightarrow Y$ a linear map. Sometimes we will say that $F$ is a version of $G$. The space of all exact sequences $0 \rightarrow Y \rightarrow \diamond \rightarrow X \rightarrow 0$ modulo equivalence can be identified with the space of all $z$-linear maps $X \curvearrowright Y$ modulo equivalence.

Definition 2.1. A $z$-linear map $F: X \curvearrowright Y$ is called $\mu$-trivial if for every $\varepsilon>0$ there is a linear map $L: X \rightarrow Y$ such that $\|F-L\| \leq \mu+\varepsilon$.

The triviality of $F$ is connected with the norm of the extension of an operator as follows:

Lemma 2.1. Given an exact sequence

$$
0 \rightarrow Y \stackrel{j}{\rightarrow} Y \oplus_{F} X \stackrel{q}{\rightarrow} X \rightarrow 0 \equiv F,
$$

a norm one operator $\tau: Y \rightarrow M$ admits an extension $T: Y \oplus_{F} X \rightarrow M$ with $\|T\| \leq \mu$ if and only if $\tau F$ is $\mu$-trivial.

Proof. If $T: Y \oplus_{F} X \rightarrow M$ is an extension of $\tau$ with $\|T\| \leq \mu$ then $L(x)=-T(0, x)$ is a linear map $X \rightarrow M$ satisfying

$$
\begin{aligned}
\|\tau F x-L x\| & =\|\tau((F x, x)-(0, x))+T(0, x)\|=\|T(F x, x)\| \\
& \leq \mu\|(F x, x)\|_{F}=\mu\|x\| .
\end{aligned}
$$

Conversely, if there is a linear map $L$ such that $\|\tau F-L\| \leq \mu$ then $T(y, x)=$ $\tau y-L x$ is a linear extension of $\tau$, which is continuous because its norm is

$$
\begin{aligned}
\|\tau y-L x\| & =\|\tau y-\tau F x+\tau F x-L x\| \leq\|\tau\|\|y-F x\|+\mu\|x\| \\
& \leq \max \{\|\tau\|, \mu\}\|(y, x)\|_{F} .
\end{aligned}
$$

In general, a $z$-linear map $F: X \curvearrowright Y$ is said to be trivial if $F \equiv 0$; this holds if and only if for some homogeneous bounded map $B: X \rightarrow Y$ and some linear map $L: X \rightarrow Y$ one has $F=B+L$. When all $z$ linear maps $X \curvearrowright Y$ are trivial, we can consider the semimetric $D(F)=$ $\operatorname{dist}(F, \operatorname{Lin}(X, Y))$ on the space of $z$-linear maps, where $\operatorname{Lin}(X, Y)$ denotes the space of linear (not necessarily continuous!) maps $X \rightarrow Y$. The following lemma can be found in [K1, CC2].

Lemma 2.2. Let $A, B$ be two Banach spaces. If $\operatorname{Ext}(A, B)=0$ then the seminorms $Z(\cdot)$ and $D(\cdot)$ are equivalent. 
A $z$-linear map has many different versions. We will need to choose good versions so that: (i) $Z(\cdot)$-convergent sequences of $z$-linear maps are pointwise convergent, and (ii) the image of a finite-dimensional space is finitedimensional. The first condition will be satisfied by the so-called canonical versions, while the second by the convexified versions. It is easy to give examples of $z$-linear maps on finite-dimensional spaces with infinite-dimensional range: define $B: \mathbb{R}^{2} \rightarrow C[0,1]$ by $B\left(e^{i \theta}\right)=x^{\theta}, 0 \leq \theta<\pi$, extended by homogeneity.

Canonical form. Let $\left(e_{\gamma}\right)$ be a Hamel basis for $X$. Given a $z$-linear map $F: X \curvearrowright Y$ we define the linear map $\ell_{F}\left(e_{\gamma}\right)=F e_{\gamma}$. The canonical form of $F$ is the $z$-linear map $\nabla F=F-\ell_{F}$, which is obviously a version of $F$. The space of all $z$-linear maps $E \curvearrowright Y$ in canonical form with respect to a given Hamel basis of $X$ will be denoted $Z_{L}(X, Y)$.

Convex form. Let $A=\left(a_{i}\right)_{i=0,1, \ldots}$ with $a_{0}=0$ be a subset of the ball of radius $1+\varepsilon$ of $X$ such that the unit ball of $X$ is contained in the closed convex hull of $A$. We define an order for finite subsets of $A:\left\{a_{i_{1}}, \ldots, a_{i_{N}}\right\} \leq$ $\left\{a_{j_{1}}, \ldots, a_{j_{M}}\right\}$ if either $N<M$, or $N=M$ and $\left\{i_{1}, \ldots, i_{N}\right\}$ is smaller than or equal to $\left\{j_{1}, \ldots, j_{N}\right\}$ in the lexicographical order. We define a homogeneous map $F^{c}: X \curvearrowright Y$ (actually it will be defined on a dense subspace of $X$, which is enough) as follows: at a point $p$ of the unit sphere of $Z$ it takes the value $F^{c}(p)=\sum_{i} \theta_{i} F a_{i}$ where $\left(a_{i}\right)_{i}$ is a minimal set for which $p$ is a convex combination $\sum_{i} \theta_{i} a_{i}$. It is clear that $\left\|F-F^{c}\right\|<Z(F)(1+\varepsilon)$ since if $\|p\|=1$ then

$$
\left\|F(p)-F^{c}(p)\right\| \leq Z(F) \sum\left|\theta_{i}\right|\left\|a_{i}\right\| .
$$

Therefore $F^{c}$ is a $z$-linear map equivalent to $F$ with $Z\left(F^{c}\right) \leq 3 Z(F)(1+\varepsilon)$. We shall say that $F^{c}$ is a convex version of $F$. It is possible to obtain a convex version of $F$ with a better estimate for its constant, but at the cost of making it more difficult to estimate the distance to $F$.

It is possible to obtain a simultaneously convex and canonical version of a $z$-linear map: taking first a linearization $F-\ell_{F}$ of $F$ with respect to a Hamel basis formed by elements with norm $1+\varepsilon$ and then convexifying with respect to a set which includes the elements of the Hamel basis. Since the convexification of a $z$-linear map with respect to a finite set of points has finite-dimensional range, one gets

Lemma 2.3. Let $F: X \curvearrowright Y$ be a z-linear map defined on a finitedimensional space. There is a version of $F$ with finite-dimensional range at a distance of $Z(F)(1+\varepsilon)$ from $F$.

Proposition 2.4. Let $F: X \curvearrowright Y$ be a z-linear map defined on a separable space. Set $X=\overline{\cup X_{n}}$ with each $X_{n}$ finite-dimensional. There is a canonical version of $F$ such that the image of each $X_{n}$ is finite-dimensional. 
Proof. One only has to choose with care the sets $A_{n} \subset X_{n}$ with respect to which the convexification is done: let first $A_{1}$ be a subset of $(1+\varepsilon) B_{X_{1}}$ such that $B_{X_{1}} \subset \operatorname{conv}\left(A_{1}\right)$. We add a $(1+\varepsilon)$-normalized basis $\left(e_{\alpha}\right)$ of $X_{1}$, and keep denoting the resulting set by $A_{1}$. The next set $A_{2} \subset X_{2}$ contains $A_{1}$, sufficiently many vectors to complete the basis of $X_{1}$ to a basis of $X_{2}$, and points with norm at most $1+\varepsilon$ so that $B_{X_{2}} \subset \operatorname{conv}\left(A_{2}\right)$. Continuing the process, we define, at each step $n$, an order on the finite subsets of $A_{n}$ compatible with the previous order for step $n-1$. Let us now consider the canonical version $G=F-\ell_{F}$ of $F$ with respect to the Hamel basis $\left(e_{\gamma}\right)$ of $\bigcup X_{n}$ just constructed, for which $\left(e_{\gamma}\right) \cap X_{k}$ is the Hamel basis we previously chose for $X_{k}$. Finally, we convexify the restrictions $G_{\left.\right|_{X_{n}}}$ with respect to the sets $A_{n}$. It is clear we get a map defined on $\bigcup X_{n}$ that, when extended to the whole $X$ (see e.g. [KP]) turns out to be a $z$-linear map in canonical form at a finite distance from $F$, and its restrictions to each $E_{n}$ have finite-dimensional range.

We will say that the final map is in convex form with respect to the sequence $\left(X_{n}\right)$.

3. The $z$-dual of a Banach space. Let $X$ be a Banach space. We define the $z$-dual of $X$ as the Banach space

$$
X^{z}=\left[Z_{L}(X, \mathbb{R}), Z(\cdot)\right] .
$$

The word "the" may seem mysterious: after all, each Hamel basis of $X$ determines, in principle, a different $z$-dual of $X$. Nevertheless one can observe that they are all isometric: indeed, if $\alpha, \mu$ are two different Hamel bases for $X$ and $\ell_{F}^{\alpha}, \ell_{F}^{\mu}$ the corresponding linear maps induced by $F$ then the correspondence $F-\ell_{F}^{\alpha} \mapsto F-\ell_{F}^{\mu}$ defines an isometry between the two $z$-duals.

The topology of pointwise convergence in $X^{z}$ will be called the $w^{*}$ topology. This is a locally convex vector topology on $X^{z}$ which, when $X$ is separable, can be made metrizable on the unit ball; it is defined by the closure operation: $F=w^{*}-\lim F_{\alpha} \Leftrightarrow \forall x \in X, F(x)=\lim F_{\alpha}(x)$. One has:

Lemma 3.1. The unit ball of $X^{z}$ is $w^{*}$-compact.

Proof. Consider the embedding $j: Z_{L}(X, \mathbb{R}) \rightarrow \mathbb{R}^{X}$ given by $j(F)=$ $(F x)_{x \in X}$. If $x=\sum_{\gamma} x_{\gamma} e_{\gamma}$ then $|F x| \leq Z(F) \sum_{\gamma}\left|x_{\gamma}\right|$, and thus $j\left(B_{Z_{L}(X, \mathbb{R})}\right)$ is contained in $\prod_{x \in X}\left[-\sum_{\gamma}\left|x_{\gamma}\right|, \sum_{\gamma}\left|x_{\gamma}\right|\right]$. That $j\left(B_{Z_{L}(X, \mathbb{R})}\right)$ is closed follows from the fact that the properties of "being $z$-linear" and "being in canonical form" are both defined by conditions compatible with pointwise convergence.

Thus, $X^{z}$ is actually a dual space, and the $w^{*}$-topology is the $w^{*}$-topology with respect to the natural predual for $X^{z}$. This can be realized as the space 
$\mathrm{co}_{z}(X)$ spanned in $\left(X^{z}\right)^{*}$ by the evaluation functionals $\delta_{x}: X^{z} \rightarrow \mathbb{R}$ given by $\delta_{x}(F)=F(x)$. We support these assertions with the following results:

Proposition 3.2. There is a $z$-linear map $\Omega_{X}: X \curvearrowright \operatorname{co}_{z}(X)$ with the property that given a z-linear map $F: X \curvearrowright Y$ there exists an operator $\phi_{F}: \operatorname{co}_{z}(X) \rightarrow Y$ such that $\phi_{F} \Omega_{X} \equiv F$. If the map $F$ is in canonical form then $\phi_{F} \Omega_{X}=F$.

Proof. We set $\Omega_{X}(x)=\delta_{x}$. This is a $z$-linear map with $Z\left(\Omega_{X}\right)=1$ since

$$
\begin{aligned}
\left\|\Omega_{X}\left(\sum x_{i}\right)-\sum \Omega_{X}\left(x_{i}\right)\right\| & =\sup _{Z(F) \leq 1}\left|\left\langle\Omega_{X}\left(\sum x_{i}\right)-\sum \Omega_{X}\left(x_{i}\right), F\right\rangle\right| \\
& =\sup _{Z(F) \leq 1}\left|F\left(\sum x_{i}\right)-\sum F\left(x_{i}\right)\right| \\
& \leq \sum\left\|x_{i}\right\| .
\end{aligned}
$$

Let $F: X \curvearrowright Y$ be in canonical form. Then $\psi_{F}(\mu)\left(y^{*}\right)=\mu\left(y^{*} F\right)$ defines an operator $\psi_{F}:\left(X^{z}\right)^{*} \rightarrow Y^{* *}$ whose restriction $\phi_{F}=\psi_{F \mid \mathrm{co}_{z}(X)}$ takes values in $Y$, which follows from

$$
\left\langle y^{*}, \phi_{F} \Omega_{X}(x)\right\rangle=\left\langle\Omega_{X}(x), y^{*} F\right\rangle=\left\langle y^{*}, F(x)\right\rangle .
$$

This also implies the equality $\phi_{F} \Omega_{X}=F$. If $F$ is not in canonical form then $\phi_{F-\ell_{F}} \Omega=F-\ell_{F}$, and we should set $\phi_{F}=\phi_{F-\ell_{F}}$.

Proposition 3.3. The map $F \mapsto \phi_{F}$ is an isometry between the Banach spaces $\left[Z_{L}(X, Y), Z(\cdot)\right]$ and $\mathfrak{L}\left(\mathrm{co}_{z}(X), Y\right)$. In particular, $X^{z}=\operatorname{co}(X)^{*}$.

Proof. Let $F: X \curvearrowright Y$ be a $z$-linear map. Then

$$
\begin{aligned}
\left\|\phi_{F}\right\| & =\sup _{\left\|\sum \lambda_{i} \Omega x_{i}\right\| \leq 1}\left\|\sum \lambda_{i} F x_{i}\right\|=\sup _{\left\|\sum \lambda_{i} \Omega x_{i}\right\| \leq 1} \sup _{\left\|y^{*}\right\| \leq 1}\left|\sum \lambda_{i} y^{*} F x_{i}\right| \\
& =\sup _{\left\|y^{*}\right\| \leq 1\left\|\sum \lambda_{i} \Omega x_{i}\right\| \leq 1}\left|\sum \lambda_{i} y^{*} F x_{i}\right|=\sup _{\left\|y^{*}\right\| \leq 1} Z\left(y^{*} F\right)=Z(F) . \text { - }
\end{aligned}
$$

Proposition 3.4. For each z-linear map $F: X \curvearrowright Y$ in canonical form there exists a z-linear map $\mathrm{co}_{z}(F): \mathrm{co}_{z}(X) \curvearrowright \mathrm{co}_{z}(Y)$ in canonical form such that $\Omega_{Y} F=\mathrm{co}_{z}(F) \Omega_{Z}$.

Proof. Just defining $\operatorname{co}_{z}(F)=\Omega_{Y} \phi_{F}$ one has

$$
\mathrm{co}_{z}(F) \Omega_{Z}=\Omega_{Y} \phi_{F} \Omega_{Z}=\Omega_{Y} F .
$$

Remark. The exact sequence $0 \rightarrow \operatorname{co}_{z}(X) \rightarrow \operatorname{co}_{z}(X) \oplus_{\Omega_{X}} X \rightarrow X \rightarrow 0$ can be viewed as a "natural projective presentation" of $X$.

In [K1] (see also [CC2]) Kalton essentially shows that if $\left(F_{n}\right)$ is a $Z(\cdot)$ convergent sequence of $z$-linear maps then the sequence of canonical versions $\nabla F_{n}$ is pointwise convergent. We now obtain what can be viewed as a converse. 
Lemma 3.5 (Change of Convergence Lemma). Let $F_{n}: X \curvearrowright Y$ be $a$ sequence of $z$-linear maps in canonical convex form on a finite-dimensional space $X$. If $F=w^{*}-\lim F_{n}$ then $F=\|\cdot\|-\lim F_{n}$.

Proof. The proof is in four steps. The first two establish the result for $\mathbb{R}$ valued maps. The third step establishes the passage from $Z(\cdot)$-convergence to norm-convergence. The fourth step yields a simple extension to finitedimensional valued maps.

SteP 1. For a metric space $K$, let $F: X \curvearrowright C(K)$ be a $z$-linear map on a finite-dimensional space having finite-dimensional range. We claim that if $p=\lim p_{n}$ then $\delta_{p} F=Z(\cdot)-\lim \delta_{p_{n}} F$.

Observe that $F\left(B_{X}\right)$ is a bounded set in a finite-dimensional space, and thus its closed convex hull is a compact, hence equicontinuous, subset of $C(K)$. We therefore have

$$
\forall \varepsilon \exists \delta>0 \forall x,\|x\| \leq 1, \forall p, q: \quad|p-q|<\delta \Rightarrow\left|\delta_{p} F x-\delta_{q} F x\right|<\varepsilon .
$$

Thus, if $\lim p_{n}=p$ then

$$
\forall \varepsilon \exists N \in \mathbb{N} \forall x,\|x\| \leq 1, \forall n>N: \quad\left|\delta_{p_{n}} F x-\delta_{p} F x\right|<\varepsilon .
$$

In this way $Z\left(\delta_{p_{n}} F-\delta_{p} F\right)=Z\left(\left(\delta_{p_{n}}-\delta_{p}\right) F\right) \leq \varepsilon$ since

$$
\left|\left(\delta_{p_{n}}-\delta_{p}\right) F\left(\sum x_{i}\right)\right|=\left|\left(\delta_{p_{n}}-\delta_{p}\right) F\left(\frac{\sum x_{i}}{\sum\left\|x_{i}\right\|}\right)\right| \sum\left\|x_{i}\right\| \leq \varepsilon \sum\left\|x_{i}\right\| .
$$

STEP 2. Let $\Delta: \operatorname{co}_{z}(X) \rightarrow C\left(B_{X^{z}}\right)$ be the canonical inclusion map. Given a $z$-linear map $F: X \curvearrowright C(K)$ in canonical form there exists an operator $\psi_{F}: C\left(B_{X^{z}}\right) \rightarrow C(K)$ such that $\psi_{F} \Delta \Omega_{X}=F$ : it is enough to take as $\psi_{F}$ the natural extension of $\phi_{F}$, namely, $\psi_{F}(f)(k)=f\left(\phi_{F}^{*}(k)\right)$. Let now $X$ be finite-dimensional and let $\Omega_{X}^{c}$ be a convexification of $\Omega_{X}$. It is clear that every $z$-linear map $F$ in convex canonical form can be written as $F=\psi_{F} \Delta \Omega_{X}^{c}$. If, moreover, $F: X \curvearrowright \mathbb{R}$ then $\psi_{F}$ is just the point evaluation $\delta_{F}$ at $F$. So, if $F_{n}$ is a collection of $z$-linear $\mathbb{R}$-valued maps in convex canonical form such that $F=w^{*}-\lim F_{n}$ then we are in the situation of the previous step and we get $\delta_{F} \Delta \Omega_{X}^{c}=Z(\cdot)-\lim \delta_{F_{n}} \Delta \Omega_{X}^{c}$, or, what is the same, $F=Z(\cdot)$ $\lim F_{n}$.

SteP 3. If $F_{n}: X \curvearrowright Y$ are $z$-linear maps in canonical form on a finitedimensional space $X$ (there is no need to require $Y$ to be finite-dimensional too) and $F=Z(\cdot)-\lim F_{n}$ then $F=\|\cdot\|-\lim F_{n}$. The result cannot be simpler: assuming $F=0$ and taking a Hamel basis $\left(e_{\gamma}\right)$ of $X$ one has

$$
\left\|F_{n}(p)\right\|=\left\|F_{n}\left(\sum p_{\gamma} e_{\gamma}\right)\right\| \leq Z\left(F_{n}\right) \sum\left|p_{\gamma}\right| \leq Z\left(F_{n}\right) \operatorname{dist}\left(E, l_{1}^{\operatorname{dim} E}\right)\|p\| .
$$

STEP 4. We now consider $z$-linear maps $F_{n}: X \curvearrowright Y$ in canonical convex form with respect to a given set $\left(a_{n}\right)$ containing a basis of $X$. One has $\sup _{n} \operatorname{dim}\left[\operatorname{Im} F_{n}\right]<+\infty$. There is a finite-dimensional space $l_{\infty}^{M}$ in which all 
the range spaces $\operatorname{Im} F_{n}$ can be placed almost isometrically. We can therefore assume that $F_{n}: X \curvearrowright l_{\infty}^{M}$. Let $\left(\delta_{j}\right)_{j=1}^{M}$ be the collection of evaluation functionals on the coordinates of $l_{\infty}^{M}$. Since $\delta_{j} F_{n}$ is in canonical convex form one has $\delta_{j} F_{n}=\delta_{\delta_{j} F_{n}} \Omega_{X}^{c}$. Moreover, if $F=w^{*}-\lim F_{n}$ then one also has $\delta_{j} F=w^{*}-\lim \delta_{j} F_{n}$ for all $1 \leq j \leq M$; thus, from the previous results one gets $\delta_{j} F=\|\cdot\|-\lim \delta_{j} F_{n}$ for all $1 \leq j \leq M$. Finally

$$
\begin{aligned}
\left\|F_{n}-F\right\| & =\sup _{\|x\| \leq 1}\left\|F_{n} x-F x\right\|=\sup _{\|x\| \leq 1} \sup _{1 \leq j \leq M}\left|\delta_{j} F_{n} x-\delta_{j} F x\right| \\
& =\sup _{1 \leq j \leq M} \sup _{\|x\| \leq 1}\left|\delta_{j} F_{n} x-\delta_{j} F x\right|=\sup _{1 \leq j \leq M}\left\|\delta_{j} F_{n}-\delta_{j} F\right\|,
\end{aligned}
$$

which is all that is needed.

4. Vector valued Sobczyk's theorem. Let us first show the equivalence between Theorems 1.2 and 1.3. Assume that Theorem 1.3 holds. Observe that a $z$-linear map $F: X \curvearrowright c_{0}\left(E_{n}\right)$ is the same as a $Z(\cdot)$-bounded sequence $F_{n}: X \curvearrowright E_{n}$ of $z$-linear maps such that $\lim \left\|F_{n} x\right\|=0$ for every $x \in X$. Indeed, if $\pi_{n}: c_{0}\left(E_{n}\right) \rightarrow E_{n}$ denotes the canonical projections then just set $F_{n}=\pi_{n} F$. For this reason we shall write $c_{0}\left(F_{n}\right)$ to denote the map $F$. Assume that we are under the hypotheses of Theorem 1.2, and have therefore an exact sequence $0 \rightarrow Y \rightarrow Z \rightarrow X \rightarrow 0 \equiv F$ with $Z(F) \leq 1$. Assume that each norm one operator $\tau_{n}$ admits an extension $\tau_{n}^{\prime}$ with $\left\|\tau_{n}^{\prime}\right\| \leq \mu$. By Lemma 2.1, the $z$-linear maps $\tau_{n} F$ are $\mu$-trivial. Since $c_{0}\left(\tau_{n}\right) F=c_{0}\left(\tau_{n} F\right)$, Theorem 1.3 asserts that $c_{0}\left(\tau_{n} F\right)$ is trivial, so it follows again from Lemma 2.1 that $c_{0}\left(\tau_{n}\right)$ admits an extension to $Y \oplus_{F} X$, hence to $E$. Conversely, if we assume that Theorem 1.2 has already been proved, Theorem 1.3 is a consequence of the construction of $\mathrm{co}_{z}(\cdot)$.

We now prove the following quantitative version of Theorem 1.3

Theorem 4.1. Let $X$ be a separable Banach space with the $\lambda$-BAP. Let $F_{n}: X \curvearrowright E_{n}$ be a sequence of $z$-linear maps with $Z\left(F_{n}\right)=1$ such that $\lim \left\|F_{n} x\right\|=0$ for every $x \in X$. If all the maps $F_{n}$ are $\mu$-trivial then the map $c_{0}\left(F_{n}\right)$ is $(\mu+2+(\mu+1) \lambda)$-trivial.

Proof. Let $X$ be a separable Banach space with the $\lambda$-BAP and let $F_{n}$ : $X \curvearrowright E_{n}$ be a sequence of $\mu$-trivial $z$-linear maps with $Z\left(F_{n}\right)=1$ and such that $\lim \left\|F_{n} x\right\|=0$ for every $x \in X$. Our purpose is to show that, for every $\varepsilon>0$, the well-defined $z$-linear map $c_{0}\left(F_{n}\right): X \curvearrowright c_{0}\left(E_{n}\right)$ is trivial.

Let $\varepsilon>0$. Let $B_{j}: X \rightarrow X$ be a sequence of finite-dimensional operators witnessing the $\lambda$-BAP of $X$; so, the sequence is pointwise convergent to the identity and $\left\|B_{j}\right\| \leq \lambda$ for each $j$. If we set $X_{1}=B_{1}(X)$ and then $X_{j+1}=B_{j+1}(X)+X_{j}$ for $j \geq 2$ we get an increasing sequence $\left(X_{j}\right)$ of finite-dimensional subspaces of $X$ such that $X=\overline{\bigcup_{j} X_{j}}$. 
We can select a canonical convex version $c_{0}\left(F_{n}\right)^{c}$ of $c_{0}\left(F_{n}\right)$ with respect to the family $\left(X_{j}\right)$. In particular, for each $j \in \mathbb{N}$, the space generated in $c_{0}\left(E_{n}\right)$ by $c_{0}\left(F_{n}\right)^{c}\left(X_{j}\right)$ is finite-dimensional; moreover, $c_{0}\left(F_{n}\right)^{c}$ vanishes on a normalized Hamel basis $\left(e_{\alpha}\right)_{\alpha}$ of $X$ compatible with the structure $\overline{U X_{j}}$ (i.e., the basis for each space contains the basis of the previous space). Also, $c_{0}\left(F_{i}\right) \equiv c_{0}\left(F_{i}\right)^{c}$ since $\left\|c_{0}\left(F_{i}\right)-c_{0}\left(F_{i}\right)^{c}\right\| \leq \sup _{i} Z\left(F_{i}\right)(1+\varepsilon)$. If $\pi_{i}$ denotes the canonical projection onto the $i$ th space, then observe that for each $i$ the $z$-linear map $\pi_{i} c_{0}\left(F_{n}\right)^{c}$ is in canonical convex form with respect to each $X_{j}$.

Since each $F_{n}$ is $\mu$-trivial there exist linear maps $L_{n}: X \rightarrow E_{n}$ such that $\left\|F_{n}-L_{n}\right\| \leq \mu$. To simplify notation we shall write $G_{i}=\pi_{i} c_{0}\left(F_{n}\right)^{c}$. We thus have a $z$-linear map $c_{0}\left(G_{n}\right) \equiv c_{0}\left(F_{n}\right)$ satisfying:

(1) Each $G_{n}$ is $(\mu+1)$-trivial.

(2) $\left(G_{n}\right)$ is pointwise convergent to 0 .

(3) Each $G_{n}$ is in canonical convex form with respect to each $X_{j}$.

(4) $c_{0}\left(G_{n}\right)$ is in canonical convex form with respect to the family $\left(X_{j}\right)$.

Our aim is to show that $c_{0}\left(G_{n}\right)$ is trivial. The change of convergence lemma 3.5 implies that for each $j \in \mathbb{N},\|\cdot\|-\lim _{n} G_{\left.n\right|_{X_{j}}}=0$, since obviously $\left(G_{n}\right)$ is pointwise convergent to 0 on each $X_{j}$. This allows us to choose for each $j$ a natural number $N(j)$ such that $\left\|G_{\left.n\right|_{X_{j}}}\right\| \leq 2^{-j}$ for each $n \geq N(j)$.

Chasing device. We are ready to construct a linear map $L: X \rightarrow$ $c_{0}\left(E_{n}\right)$ at a finite distance from $c_{0}\left(G_{n}\right)$ as follows: we set $L(x)(n)=L_{n}(x)$ for $n \leq N(1)-1$, and

$$
L(x)(n)=\left(L_{n}-L_{n} \circ B_{j}\right)(x) \quad \text { for } N(j) \leq n<N(j+1) .
$$

We show first that $L(x) \in c_{0}\left(E_{n}\right)$ for each $x \in \bigcup_{n=1}^{\infty} X_{n}$. Indeed, if $x \in \bigcup_{n=1}^{\infty} X_{n}$ then there exists $j$ such that $x \in X_{j}$; thus, for $n \geq N(j)+1$, $\|L(x)(n)\|=\left\|L_{n}(x)-L_{n} \circ B_{j}(x)\right\|=\left\|L_{n}\left(x-B_{j}(x)\right)\right\| \leq\left\|L_{n \mid X_{j}}\right\|\left\|x-B_{j} x\right\|$. The argument concludes by taking into account that $\lim _{j}\left\|x-B_{j} x\right\|=0$ and

$$
\left\|L_{\left.n\right|_{X_{j}}}\right\| \leq\left\|G_{\left.n\right|_{X_{j}}}-L_{\left.n\right|_{X_{j}}}\right\|+\left\|G_{\left.n\right|_{X_{j}}}\right\| \leq \mu+1+2^{-j} .
$$

Finally, on the dense subspace $\bigcup_{n=1}^{\infty} X_{n}$ of $X$ the map $c_{0}\left(G_{n}\right)$ is at a finite distance from $L$ :

$$
\begin{aligned}
\left\|c_{0}\left(G_{n}\right)-L\right\| & =\sup _{n}\left\|G_{n}-\pi_{n} L\right\| \\
& \leq \sup _{n}\left(\left\|G_{n}-L_{n}\right\|+\left\|L_{\left.n\right|_{E_{j}}} B_{j}\right\|\right) \leq \mu+1+\left(\mu+1+2^{-j}\right) \lambda .
\end{aligned}
$$

Folklore extension results (see e.g. [KP]) imply that $c_{0}\left(G_{n}\right)$ must be equally trivial. Therefore, $c_{0}\left(F_{n}\right)$ will be $(\mu+2+(\mu+1) \lambda)$-trivial.

We now prove the necessity of the BAP hypothesis. 
Proposition 4.2. Let $X$ be a separable Banach space. Then $X$ has the $B A P$ if and only if whenever one has an exact sequence $0 \rightarrow Y \rightarrow Z \rightarrow$ $X \rightarrow 0$ and a SOT-null sequence of norm one operators $t_{n}: Y \rightarrow E_{n}$, each admitting an extension $T_{n}: Z \rightarrow E_{n}$ with sup $\left\|T_{n}\right\|<+\infty$, then the map $c_{0}\left(t_{n}\right): Y \rightarrow c_{0}\left(E_{n}\right)$ can be extended to $Z$.

Proof. The "only if" part has already been proved. Let $X$ be a separable space represented as $X=\overline{\bigcup_{n} X_{n}}$ with each $X_{n}$ finite-dimensional. Set $c\left(X_{n}\right)=\left\{\left(x_{n}\right): x_{n} \in X_{n}, \lim x_{n}\right.$ exists $\}$ (see [JO]). The exact sequence

$$
0 \rightarrow c_{0}\left(X_{n}\right) \rightarrow c\left(X_{n}\right) \stackrel{\lim \cdot}{\longrightarrow} X \rightarrow 0
$$

splits if and only if $X$ has the BAP. Indeed, since $c\left(X_{n}\right)$ has a monotone FDD, if the sequence splits, $X$ must have the BAP. If, on the other hand, $X$ has a sequence $\left(B_{n}\right)$ of finite rank operators pointwise convergent to the identity, since there is no loss of generality in assuming that $B_{n}(X) \subset X_{n}$, it turns out that the operator $S(x)=\left(B_{n} x\right)_{n}$ defines a continuous linear selection for the quotient map $\lim$. Now, if $X$ does not enjoy the BAP then $c_{0}\left(X_{n}\right)$ cannot be complemented in $c\left(X_{n}\right)$ and the sequence does not split. Let $\pi_{n}: c_{0}\left(X_{n}\right) \rightarrow X_{n}$ be the natural projection. It admits a norm one extension given by the natural projection $\pi_{n}: c\left(X_{n}\right) \rightarrow X_{n}$. However, $c_{0}\left(\pi_{n}\right)$ is the identity of $c_{0}\left(X_{n}\right)$, which cannot be extended.

A modification of the chasing device yields the following version for separably injective spaces. Observe that no BAP is now required.

Theorem 4.3. Let $X$ be a separable Banach space and let $\left(E_{n}\right)$ be a sequence of $\mu$-separably injective Banach spaces. Let $F_{n}: X \curvearrowright E_{n}$ be a sequence of $z$-linear maps with $Z\left(F_{n}\right)=1$ such that $\lim \left\|F_{n} x\right\|=0$ for every $x \in X$. Then the map $c_{0}\left(F_{n}\right)$ is $(2 \mu+2)$-trivial.

Proof. The proof goes as before with the following modifications. That each $F_{n}: X \curvearrowright E_{n}$ is $\mu$-trivial follows from the $\mu$-separable injectivity of $E_{n}$. The canonical convex forms $G_{n}$ therefore admit linear maps $L_{n}: X \rightarrow E_{n}$ such that $\left\|G_{n}-L_{n}\right\| \leq \mu+1$. It only remains to see how to define the linear map $L: X \rightarrow c_{0}\left(E_{n}\right)$ at a finite distance from $c_{0}\left(G_{n}\right)$. To do that we just make a slight modification in the chasing device: Let $\widehat{L}_{\left.n\right|_{X_{j}}}$ be a continuous linear extension of the restriction $L_{\left.n\right|_{X_{j}}}$ provided by the $\mu$-separable injectivity of $E_{n}$. Define $L$ by

$$
L(x)(n)=\left(L_{n}-\widehat{L}_{\left.n\right|_{X_{j}}}\right)(x) \quad \text { if } N(j) \leq n<N(j+1)
$$

(we can set $L(x)(n)=L_{n}(x)$ for $n \leq N(1)-1$ ). As before, it is enough to verify that $L x \in c_{0}\left(X_{n}\right)$ and $\left\|c_{0}\left(G_{n}\right)-L\right\|$ is (uniformly) finite on the dense part $\bigcup_{n=1}^{\infty} X_{n}$ of $X$. That $L$ is well defined is easy: if $x \in \bigcup_{n=1}^{\infty} X_{n}$, there exists $j$ such that $x \in X_{j}$; thus, $L(x)(n)=0$ for all $n \geq N(j)$. That $L$ is at 
a finite distance from $c_{0}\left(G_{n}\right)$ follows from the estimate

$$
\left\|L_{\left.n\right|_{X_{j}}}\right\| \leq\left\|G_{\left.n\right|_{X_{j}}}-L_{\left.n\right|_{X_{j}}}\right\|+\left\|G_{\left.n\right|_{X_{j}}}\right\| \leq \mu+1+2^{-j},
$$

which yields

$\left\|c_{0}\left(G_{n}\right)-L\right\|=\sup _{n}\left\|G_{n}-\pi_{n} L\right\| \leq \sup _{n}\left\|G_{n}-L_{n}\right\|+\left\|L_{\left.n\right|_{X_{j}}}\right\| \leq 2 \mu+1+2^{-j}$.

It follows that $c_{0}\left(F_{n}\right)$ is $(2 \mu+2)$-trivial.

When the spaces $E_{n}$ are the scalar field, which is 1-separably injective, the convexification process is no longer needed, and thus one gets

Corollary 4.1 (Sobczyk's theorem). Each z-linear map $F: X \curvearrowright c_{0}$ defined on a separable Banach space $X$ is 2-trivial.

Proof. The only modification to make in the chasing device is to approach $L_{n}$ with a Hahn-Banach extension $\widehat{L}_{\left.n\right|_{X_{j}}}$ of its restriction $L_{\left.n\right|_{X_{j}}}$.

A further version for a different combination between the properties of the target spaces and the quotient space will be presented in Proposition 5.3 .

Quantitative estimates, revisited. As we have seen, the quality of the estimate depends on the combination between properties of the quotient space $X$ and the target space $E$. The estimate worsens when one translates the nonlinear approach to an extension result for operators, since Lemma 2.1 multiplies the estimate by 3; another deterioration occurs when a passage to the canonical or convex form of a $z$-linear map is necessary. Theorem 4.1 thus becomes:

THEOREM 4.4. Let $Y$ be a subspace of a Banach space $Z$ such that $Z / Y$ is separable and has the $\lambda$-BAP. Let $\tau=\left(\tau_{n}\right): Y \rightarrow c_{0}\left(E_{n}\right)$ be an operator. If each $\tau_{n}$ admits an extension $T_{n}: Z \rightarrow E_{n}$ with $\left\|T_{n}\right\| \leq \mu\left\|\tau_{n}\right\|$ then there is an extension $T: Z \rightarrow c_{0}\left(E_{n}\right)$ of $\tau$ with $\|T\| \leq 3(\mu+2+(\mu+1) \lambda)\|\tau\|$.

While Theorem 4.3 becomes

THEOREM 4.5. If $\left(E_{n}\right)$ is a sequence of $\mu$-separably injective Banach spaces then $c_{0}\left(E_{n}\right)$ is $(6 \mu+6)$-separably injective.

\section{Applications}

5.1. Nonlinear estimates. Working in nonlinear terms is somehow simpler. The introduction of the following (possibly infinite) parameter will simplify the exposition:

$$
z(B, A)=\inf \{\lambda \geq 0: D(\cdot) \leq \lambda Z(\cdot)\}
$$

where the infimum is taken over all $z$-linear maps $F: B \curvearrowright A$. By Lemma 2.2 . $\operatorname{Ext}(B, A)=0$ if and only if $z(B, A)<+\infty$. Theorem 4.1 becomes: 
Corollary 5.1. Let $X$ be a separable Banach space with the $\lambda$-BAP. If $\left(E_{n}\right)$ is a sequence of Banach spaces such that $\sup z\left(X, E_{n}\right) \leq \mu$ then $z\left(X, c_{0}\left(E_{n}\right)\right) \leq \mu+\mu \lambda$.

A nice version of Sobczyk's theorem is as follows:

Corollary 5.2. Let $X$ be a separable Banach space. If $\left(E_{n}\right)$ is a sequence of $\mathcal{L}_{\infty, \lambda}$-spaces then

$$
z\left(X, c_{0}\left(E_{n}\right)\right) \leq 2 \lambda \sup _{n} z\left(X, E_{n}\right) .
$$

The proof is immediate by now: just observe how the chasing device works because the $\mathcal{L}_{\infty, \lambda}$ character of the space guarantees extension with norm at most $\lambda$ of finite-dimensional operators (in our case, of $L_{\left.n\right|_{E_{j}}}$ ).

In [CCKY, Theorem 4.1], the following crucial characterization of the spaces $X$ for which $\operatorname{Ext}\left(X, C\left(\omega^{\omega}\right)\right)=0$ is presented:

Corollary 5.3 (Cabello-Castillo-Kalton-Yost [CCKY]). Suppose $X$ is a separable Banach space. Then $\operatorname{Ext}\left(X, C\left(\omega^{\omega}\right)\right)=0$ if and only if

$$
\sup _{n} \pi_{n}(X)<+\infty \text {. }
$$

The parameter $\pi_{n}(X)$, introduced in [CCKY], Section 3], is easily checked to be $\pi_{n}(X)=z\left(X, C\left(\omega^{n}\right)\right)$. Now, since $c_{0}\left(C\left(\omega^{n}\right)\right)$ is isomorphic to a hyperplane of $C\left(\omega^{\omega}\right)$ one has

$$
z\left(X, C\left(\omega^{\omega}\right)\right) \leq 2 \sup _{n} z\left(X, C\left(\omega^{n}\right)\right) .
$$

Corollary 5.4. Let $\left(E_{n}\right)$ be a sequence of spaces $\mu$-complemented in their biduals and let $X$ be a separable $\mathcal{L}_{1, \lambda}$-space. Then $z\left(X, c_{0}\left(E_{n}\right)\right) \leq \mu+$ $\mu \lambda$. In particular $\operatorname{Ext}\left(X, c_{0}\left(E_{n}\right)\right)=0$.

The following particular case is especially interesting:

Corollary 5.5. Let $H$ be a subspace of $c_{0}$ and let $X$ be a separable $\mathcal{L}_{1, \lambda}$-space. Then $\operatorname{Ext}(X, H)=0$.

Proof. Recall that $z(X, A) \leq \lambda$ for $A$ finite-dimensional and $X$ an $\mathcal{L}_{1, \lambda^{-}}$ space. Therefore, for every sequence $\left(A_{n}\right)$ of finite-dimensional spaces,

$$
z\left(X, c_{0}\left(A_{n}\right)\right) \leq 1+\lambda<+\infty .
$$

General structure results of Johnson-Rosenthal and Zippin (see [LT, 1.g.2 and 2.d.1]) imply that given a subspace $H$ of $c_{0}$ there exist sequences $\left(A_{n}\right)$ and $\left(B_{n}\right)$ of finite-dimensional spaces such that there is an exact sequence $0 \rightarrow c_{0}\left(A_{n}\right) \rightarrow H \rightarrow c_{0}\left(B_{n}\right) \rightarrow 0$. Since $\operatorname{Ext}\left(X, c_{0}\left(A_{n}\right)\right)=0=\operatorname{Ext}\left(X, c_{0}\left(B_{n}\right)\right)$ $=0$, it immediately follows that $\operatorname{Ext}(X, H)=0$. 
5.2. Lifting results. In situations involving the canonical embedding $c_{0}\left(E_{n}\right) \rightarrow l_{\infty}\left(E_{n}\right)$ it is natural to apply a result that includes among its hypotheses the uniformly bounded extension of a sequence of operators pointwise convergent to zero. That is the content of the following lemma.

Lemma 5.1. Let $\left(E_{n}\right)$ be any sequence of Banach spaces and let $X$ be a separable Banach space with the BAP. Then every operator $T: X \rightarrow$ $l_{\infty}\left(E_{n}\right) / c_{0}\left(E_{n}\right)$ can be lifted to an operator $X \rightarrow l_{\infty}\left(E_{n}\right)$.

Proof. Let $q_{X}: l_{1} \rightarrow X$ be a quotient map. Observe the commutative diagram

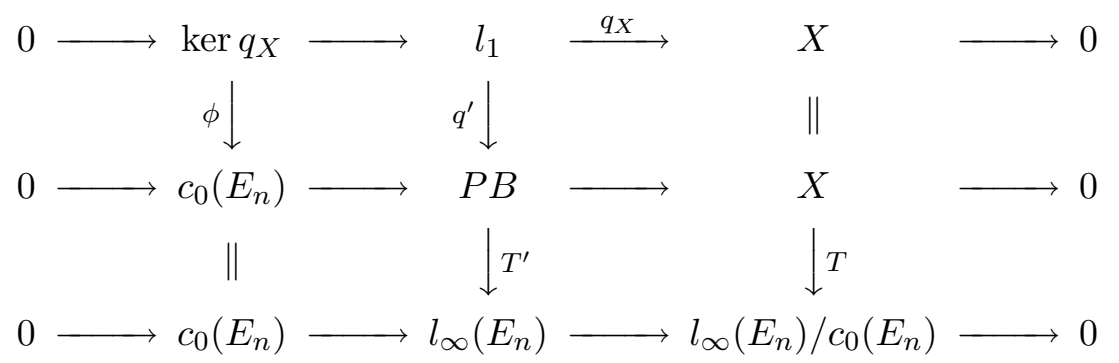

in which $q^{\prime}$ is a lifting of $q_{X}$ to the pull-back space $P B$, and $\phi$ is the restriction of $q^{\prime}$ to $\operatorname{ker} q_{X}$. The operator $\phi$ satisfies the hypothesis of Theorem 1.2 since $T^{\prime} q^{\prime}$ yields the uniformly bounded sequence of extensions of the operators defining $\phi$. Since $X$ has the BAP, an extension $l_{1} \rightarrow c_{0}\left(E_{n}\right)$ of $\phi$ exists. The upper part of the above diagram is also a push-out diagram, hence the sequence

$$
0 \rightarrow c_{0}\left(E_{n}\right) \rightarrow P B \rightarrow X \rightarrow 0
$$

splits; and since it is also a pull-back sequence, $T$ can be lifted to an operator $X \rightarrow l_{\infty}\left(E_{n}\right)$.

Given a sequence $\left(E_{n}\right)$ of Banach spaces let $Q\left[E_{n}\right]$ denote the space $l_{\infty}\left(E_{n}\right) / c_{0}\left(E_{n}\right)$. If all $E_{n}=E$ then we simply write $Q[E]$. The next result asserts that regarding the vanishing of Ext, the spaces $c_{0}\left(E_{n}\right)$ and $Q\left[E_{n}\right]$ behave similarly. More precisely:

Proposition 5.2. If $X$ is a separable Banach space with the BAP, and $E$ a Banach space such that $\operatorname{Ext}(X, E)=0$, then $\operatorname{Ext}(X, Q[E]))=0$.

Proof. Let $0 \rightarrow Q[E] \rightarrow M \rightarrow X \rightarrow 0$ be an exact sequence. Combining a projective presentation for $X$ with the exact sequence $0 \rightarrow c_{0}(E) \rightarrow$ $l_{\infty}(E) \rightarrow Q[E] \rightarrow 0$ in a commutative diagram we get 


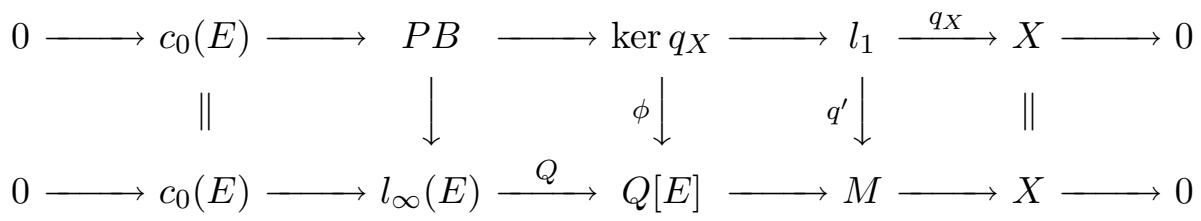

Since every space with the BAP is a complemented subspace of a space with basis, if $X$ has the BAP then $X \oplus F$ has a basis for some space $F$. So we know from Lusky [L1, L2] that the kernel of a quotient map $q$ : $l_{1} \rightarrow X \oplus F$ has a basis. Let $q_{F}: l_{1} \rightarrow F$ be a quotient map. The operator $q_{X} \oplus q_{F}: l_{1} \oplus l_{1} \rightarrow X \oplus F$ is such a quotient map, hence its kernel $\operatorname{ker}\left(q_{X} \oplus q_{F}\right) \simeq \operatorname{ker} q_{X} \oplus \operatorname{ker} q_{F}$ has a basis and therefore ker $q_{X}$ has the BAP. From Lemma 5.1 it follows that the operator $\phi$ can be lifted to an operator $\Psi: \operatorname{ker} q_{X} \rightarrow l_{\infty}(E)$. On the other hand, since $\operatorname{Ext}(X, E)=0$, we have $\operatorname{Ext}\left(X, l_{\infty}(E)\right)=0$; therefore $\Psi$ can be extended to an operator $\Phi: l_{1} \rightarrow l_{\infty}(E)$. The operator $Q \Phi$ is the extension of $\phi$ which shows that the initial exact sequence splits.

Corollary 5.6. If $E$ is separably injective then $Q[E]$ is separably injective.

5.3. Vector sums of Lindenstrauss-Pełczyński spaces. In [CMS], the class of all $\mathcal{L}_{\infty}$-spaces satisfying the Lindenstrauss-Pełczyński theorem [LP was isolated. More precisely, a Banach space $X$ is said to be an $\mathcal{L P}_{\lambda^{-}}$ space if every norm one operator from a subspace of $c_{0}$ into $X$ can be extended to an operator on the whole $c_{0}$ with norm at most $\lambda$. A Banach space is said to be a Lindenstrauss-Petczyński space, for short an $\mathcal{L} \mathcal{P}$-space, if it is an $\mathcal{L} \mathcal{P}_{\lambda}$-space for some $\lambda \geq 1$.

Proposition 5.3. Let $\left(Y_{n}\right)_{n}$ be a sequence of $\mathcal{L} \mathcal{P}_{\lambda}$-spaces with $\lambda \geq 1$. Then the $c_{0}$-vector sum $c_{0}\left(Y_{n}\right)$ is an $\mathcal{L P}_{\lambda+\lambda^{2}}$-space.

Proof. Let us consider $0 \rightarrow H \rightarrow c_{0} \rightarrow c_{0} / H \rightarrow 0 \equiv F$ and an operator $T: H \rightarrow c_{0}\left(Y_{n}\right)$. For every $n$, let $\pi_{n}: c_{0}\left(Y_{n}\right) \rightarrow Y_{n}$ be the natural projection and let $T_{n}=\pi_{n} T$. So it makes sense to write $T=c_{0}\left(T_{n}\right)$. We can assume $H=\overline{\bigcup_{j} H_{j}}$ for some increasing sequence $\left(H_{j}\right)_{j}$ of finite-dimensional spaces. It is not hard to prove (this was already done in $z$-linear terms with the convexification process) that for every $j \in \mathbb{N}$ we can get a commutative diagram

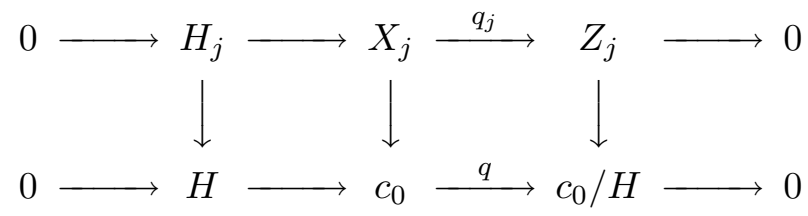


where vertical arrows are embeddings and $\left(Z_{j}\right)_{j}$ is an increasing sequence of finite-dimensional spaces such that $c_{0} / H=\overline{\bigcup_{j} Z_{j}}$ and $c_{0}=\overline{\bigcup_{j} X_{j}}$.

Since $\lim _{n}\left\|T_{n}(h)\right\|=0$ for every $h \in H$ and $H_{j}$ is finite-dimensional, the sequence $\left(T_{n, j}\right)_{n}$ formed by the restrictions of $T_{n}$ to $H_{j}$ converges in norm to 0 . Let $v(j)$ be a natural number such that $\left\|T_{n, j}\right\| \leq 2^{-j}$ for $n \geq v(j)$. Let $\widehat{T}_{n}: c_{0} \rightarrow Y_{n}$ be an extension of $T_{n}$ with $\left\|\widehat{T}_{n}\right\| \leq \lambda\left\|T_{n}\right\|$, which exists by hypothesis. Let $\widehat{T}_{n, j}: c_{0} \rightarrow Y_{n}$ be an extension of $T_{n, j}$ such that $\left\|\widehat{T}_{n, j}\right\| \leq$ $\lambda\left\|T_{n, j}\right\|$, which, once again, exists by hypothesis. Since $\widehat{T}_{n}-\widehat{T}_{n, j}$ vanishes on $H_{j}$ there is an operator $\phi_{n, j}: Z_{j} \rightarrow Y_{n}$ such that $\widehat{T}_{n}-\widehat{T}_{n, j}=\phi_{n, j} q_{j}$. But $\phi_{n, j}$ admits an extension $\widehat{\phi}_{n, j}: c_{0} / H \rightarrow Y_{n}$ with $\left\|\widehat{\phi}_{n, j}\right\| \leq \lambda\left\|\phi_{n, j}\right\|$ since $c_{0} / H$ is a subspace of $c_{0}$. We now define an operator $\psi: c_{0} \rightarrow c_{0}\left(Y_{n}\right)$ as follows: for any $x \in c_{0}$,

$$
\psi x(n)=\widehat{T}_{n} x-\widehat{T}_{n, j} x+\widehat{\phi}_{n, j} q x, \quad v(j) \leq n<v(j+1),
$$

and 0 for $n<v(1)$. Let us see that $\psi$ is the desired extension of $T$ :

(1) To check $\psi$ takes values in $c_{0}\left(Y_{n}\right)$ it is sufficient to do it on $\bigcup_{n} X_{n}$ : If $x \in \bigcup X_{n}$ then $x \in X_{j}$ for some $j$; consequently, $\widehat{T}_{n} x-\widehat{T}_{n, j} x+$ $\widehat{\phi}_{n, j} q(x)=0$ for every $n \geq j$.

(2) To estimate $\|\psi\|$ it is enough to do it in each coordinate:

$$
\|\psi x(n)\| \leq\left\|\widehat{T}_{n}-\widehat{T}_{n, j}\right\|+\left\|\widehat{\phi}_{n, j} q\right\| \leq \lambda+\lambda^{2}+2 \lambda 2^{-j} .
$$

The following problem was posed in [CMS].

Problem 5.7. Is $l_{\infty} / C[0,1]$ an $\mathcal{L} \mathcal{P}$-space?

We provide a partial answer:

Corollary 5.8. If $E$ is an $\mathcal{L} \mathcal{P}$-space then $Q[E]$ is an $\mathcal{L} \mathcal{P}$-space.

Proof. By the result of Johnson-Rosenthal-Zippin (see [LT, 1.g.2 and 2.d.1]) and the general techniques used in [CMS] it is enough to work with subspaces of $c_{0}$ having the form $H=c_{0}\left(F_{n}\right)$ for finite-dimensional spaces $F_{n}$. These $H$ obviously have the BAP. Every operator $t: H \rightarrow Q[E]$ can therefore be lifted to an operator $t_{1}: H \rightarrow l_{\infty}(E)$, and then extended to an operator $T: c_{0} \rightarrow l_{\infty}(E)$. If $Q: l_{\infty}(E) \rightarrow Q[E]$ is the natural quotient map then $Q T$ is the desired extension of $t$.

Acknowledgements. This research has been partially supported by Project MTM2007-67994-C02-02. Thanks are due to Félix Cabello for pointing out the necessity in Proposition 4.2. The work of the referee to improve the quality of the exposition of the paper is gratefully acknowledged. 


\section{References}

[ACGJM] S. A. Argyros, J. M. F. Castillo, A. S. Granero, M. Jimenez and J. P. Moreno, Complementation and embeddings of $c_{0}(I)$ in Banach spaces, Proc. London Math. Soc. 85 (2002), 742-772.

[ACCGM] A. Avilés, F. Cabello, J. M. F. Castillo, M. González and Y. Moreno, On separably injective Banach spaces, preprint, 2010.

[C] F. Cabello Sánchez, Yet another proof of Sobczyk's theorem, in: Methods in Banach Space Theory (Caceres, 2004), J. M. F. Castillo and W. B. Johnson (eds.), London Math. Soc. Lecture Note Ser. 337, Cambridge Univ. Press, 2006, 136-138.

[CC1] F. Cabello Sánchez and J. M. F. Castillo, Duality and twisted sums of Banach spaces, J. Funct. Anal. 175 (2000), 1-16.

[CC2] - - - Uniform boundedness and twisted sums of Banach spaces, Houston J. Math. 30 (2004), 523-536.

[CCKY] F. Cabello Sánchez, J. M. F. Castillo, N. J. Kalton and D. T. Yost, Twisted sums with $C(K)$ spaces, Trans. Amer. Math. Soc. 355 (2003), 4523-4541.

[CCY] F. Cabello Sánchez, J. M. F. Castillo and D. T. Yost, Sobczyk's theorem from A to B, Extracta Math. 15 (2000), 391-420.

[CA] J. M. F. Castillo, Banach spaces, à la recherche du temps perdu, ibid. 15 (2000), 291-334.

[CG] J. M. F. Castillo and M. González, Three-Space Problems in Banach Space Theory, Lecture Notes in Math. 1667, Springer, 1997.

[CM] J. M. F. Castillo and Y. Moreno, On the Lindenstrauss-Rosenthal theorem, Israel J. Math. 140 (2004), 253-270.

[CMS] J. M. F. Castillo, Y. Moreno and J. Suárez, On Lindenstrauss-Pełczyński spaces, Studia Math. 174 (2006), 213-231.

[GP] E. Galego and A. Plichko, On Banach spaces containing complemented and uncomplemented subspaces isomorphic to $c_{0}$, Extracta Math. 18 (2003), 315319 .

[J] W. B. Johnson, Extensions of $c_{0}$, Positivity 1 (1997), 55-74.

[JO] W. B. Johnson and T. Oikhberg, Separable lifting property and extensions of local reflexivity, Illinois J. Math. 45 (2001) 123-137.

[JZ] W. B. Johnson and M. Zippin, Extension of operators from weak ${ }^{*}$-closed subspaces of $l_{1}$ into $C(K)$ spaces, Studia Math. 117 (1995), 43-55.

[K1] N. J. Kalton, The three-space problem for locally bounded F-spaces, Compos. Math. 37 (1978), 243-276.

[K2] - Locally complemented subspaces and $\mathcal{L}_{p}$ spaces for $0<p<1$, Math. Nachr. 115 (1984), 71-97.

[KP] N. J. Kalton and N. T. Peck, Twisted sums of sequence spaces and the three space problem, Trans. Amer. Math. Soc. 255 (1979), 1-30.

[KZ] P. Koszmider and P. Zieliński, Complementation and decompositions in some weakly Lindelöf Banach spaces, preprint, 2010.

[LP] J. Lindenstrauss and A. Pełczyński, Contributions to the theory of the classical Banach spaces, J. Funct. Anal. 8 (1971), 225-249.

[LT] J. Lindenstrauss and L. Tzafriri, Classical Banach Spaces I. Sequence Spaces, Ergeb. Math. Grenzgeb. 92, Springer, 1977.

[L1] W. Lusky, Three-space problems and basis extensions, Israel J. Math. 107 (1988), 17-27. 
[L2] W. Lusky, Three-space problems and bounded approximation property, Studia Math. 159 (2003), 417-434.

[R] H. P. Rosenthal, The complete separable extension problem, J. Operator Theory 43 (2000), 329-374.

[S] A. Sobczyk, On the extension of linear transformations, Trans. Amer. Math. Soc. 55 (1944), 153-169.

Jesús M. F. Castillo, Yolanda Moreno

Departamento de Matemáticas

Universidad de Extremadura

Avenida de Elvas

06071 Badajoz, Spain

E-mail: castillo@unex.es

ymoreno@unex.es

Received October 12, 2009

Revised version August 28, 2010 